\title{
Impaired efferocytosis and neutrophil extracellular trap clearance by macrophages in ARDS
}

\author{
Murielle Grégoire ${ }^{1,2}$, Fabrice Uhel ${ }^{2,3,4}$, Mathieu Lesouhaitier ${ }^{3,4}$, \\ Arnaud Gacouin $\mathbb{1}^{3,4}$, Marion Guirriec ${ }^{1,2}$, Frederic Mourcin ${ }^{1,2}$, \\ Erwan Dumontet ${ }^{1,2}$, Arnaud Chalin ${ }^{5}$, Michel Samson ${ }^{5}$, Laure-Line Berthelot ${ }^{6}$, \\ Adrien Tissot ${ }^{6,7}$, Mallorie Kerjouan ${ }^{8}$, Stéphane Jouneau ${ }^{5,8}$, Yves Le Tulzo ${ }^{2,3,4}$, \\ Karin Tarte ${ }^{1,2}$, Jaroslaw W. Zmijewski ${ }^{9}$ and Jean-Marc Tadié $2,3,4$
}

Affiliations: ${ }^{1} \mathrm{CHU}$ Rennes, Pôle Biologie, Rennes, France. ${ }^{2}$ Université Rennes 1, INSERM, EFS Bretagne, UMR U1236, Rennes, France. ${ }^{3} \mathrm{CHU}$ Rennes, Maladies Infectieuses et Réanimation Médicale, Rennes, France. ${ }^{4}$ Inserm CIC-1414, Faculté de Médecine, Université Rennes 1, Rennes, France. ${ }^{5}$ Université Rennes 1, INSERM UMR U1085, Institut de Recherche en Santé, Environnement et Travail (IRSET). Rennes, France. 'Université de Nantes, INSERM, UMR_S 1064, Centre de Recherche en Transplantation et Immunologie (CRTI), Nantes, France. ${ }^{7} \mathrm{CHU}$ Nantes, Service de Pneumologie, Nantes, France. ${ }^{8} \mathrm{CHU}$ Rennes, Service de Pneumologie, Rennes, France. ${ }^{9}$ Dept of Medicine, University of Alabama at Birmingham, Birmingham, AL, USA.

Correspondence: Jean-Marc Tadié, Service des Maladies Infectieuses et Réanimation Médicale, Hôpital Pontchaillou, 2 rue Henri Le Guilloux, 35033 Rennes Cedex 9, France. E-mail: jeanmarc.tadieAachu-rennes.fr

@ERSpublications

Restoration of AMPK activation and specific inhibition of HMGB1 could reduce lung inflammation during human ARDS http://ow.ly/bxCj30ktyiZ

Cite this article as: Grégoire M, Uhel F, Lesouhaitier M, et al. Impaired efferocytosis and neutrophil extracellular trap clearance by macrophages in ARDS. Eur Respir J 2018; 52: 1702590 [https://doi.org/ 10.1183/13993003.02590-2017].

ABSTRACT Exaggerated release of neutrophil extracellular traps (NETs) along with decreased NET clearance and inability to remove apoptotic cells (efferocytosis) may contribute to sustained inflammation in acute respiratory distress syndrome (ARDS). Recent studies in experimental models of ARDS have revealed the crosstalk between AMP-activated protein kinase (AMPK) and high-mobility group box 1 (HMGB1), which may contribute to effectiveness of efferocytosis, thereby reducing inflammation and ARDS severity.

We investigated neutrophil and NET clearance by macrophages from control and ARDS patients and examined how bronchoalveolar lavage (BAL) fluid from control and ARDS patients could affect NET formation and efferocytosis. Metformin (an AMPK activator) and neutralising antibody against HMGB1 were applied to improve efferocytosis and NET clearance.

Neutrophils from ARDS patients showed significantly reduced apoptosis. Conversely, NET formation was significantly enhanced in ARDS patients. Exposure of neutrophils to ARDS BAL fluid promoted NET production, while control BAL fluid had no effect. Macrophage engulfment of NETs and apoptotic neutrophils was diminished in ARDS patients. Notably, activation of AMPK in macrophages or neutralisation of HMGB1 in BAL fluid improved efferocytosis and NET clearance.

In conclusion, restoration of AMPK activity with metformin or specific neutralisation of HMGB1 in BAL fluid represent promising therapeutic strategies to decrease sustained lung inflammation during ARDS. 


\section{Introduction}

Acute respiratory distress syndrome (ARDS) is an acute inflammatory lung injury characterised by a hypoxaemic respiratory failure following a disruption of the endothelial-epithelial barrier, alveolar damage and pulmonary oedema [1,2]. In spite of significant advances in critical care, antibiotics and lung ventilation strategies, effective therapeutic interventions to diminish the severity of lung injury and mortality among ARDS patients are not available [3-5]. Neutrophils are the first line of innate immune response, producing antibacterial peptides, reactive oxygen species, cytokines and other inflammatory mediators [6]. Neutrophils are also able to release neutrophil extracellular traps (NETs), a unique mechanism of DNA deployment into the extracellular milieu [7,8]. Although these functions are important to target microbial agents, exaggerated and prolonged activation of neutrophils could contribute to the development of acute lung injury (ALI) [9-11]. In particular, the lifespan of neutrophils is prolonged during ARDS and several studies have shown a deleterious impact associated with delayed apoptosis of neutrophils [9, 12-14]. Similar to substantial production of inflammatory mediators, neutrophil-driven excessive NET formation can worsen inflammation, in particular in sterile inflammatory conditions [15-17]. Therefore, time-dependent neutralisation of apoptotic cells, especially apoptotic neutrophils, and clearance of NETs have appeared to be important steps in the resolution phase and recovery from lung injury, since effective removal of dying cells (known as efferocytosis) plays a crucial role in the maintenance of tissue homeostasis [18]. Macrophage phagocytic function is typically associated with engulfment of dying cells; however, less is known about the mechanisms involved in NET clearance [19-21]. Besides the recently described benefit of DNase I in experimental sepsis, the role of macrophages in the clearance of NETs, including in conditions associated with development and resolution of ARDS, has not been determined [22].

AMP-activated protein kinase (AMPK) is a serine-threonine protein kinase that functions as a crucial metabolic sensor and regulates cellular energy production and expenditure [23]. Recent studies indicate that AMPK activation also has a potent anti-inflammatory effect. In addition, AMPK activation can stimulate macrophage efferocytosis, along with neutrophil and macrophage capacity to ingest bacteria [24, 25]. However, inflammatory conditions are accompanied by a reduced activity of AMPK in macrophages, in neutrophils and in lung tissue. Restoration of AMPK activity could be an interesting approach to increase efferocytosis and would be likely to decrease inflammatory lung injury in humans, as already reported in mouse models of ALI $[25,26]$. Moreover, high-mobility group box 1 (HMGB1), an alarmin that may promote inflammation, has been involved in the development of severe ARDS and has been shown to inhibit efferocytosis [27-29].

We thus designed the present study to investigate the ability of neutrophils and macrophages to regulate lung inflammation in patients with ARDS. Our first objectives were to evaluate the survival of neutrophils and their ability to produce NETs. Secondly, we studied macrophage capacity to engulf apoptotic cells and NETs. Finally, two potential therapeutic targets, AMPK and HMGB1, were investigated for their ability to restore efferocytosis and NET clearance, and thus to reduce persistent inflammation and decrease lung injury in patients with ARDS.

\section{Materials and methods \\ Patients}

This study was conducted in the medical intensive care unit (ICU) of Rennes University Hospital (Rennes, France). The study protocol was approved by the local ethics committee (number 14.38). Because of the observational nature of the study, a non-opposition form was provided to families and patients. Patients with the Berlin criteria for ARDS were consecutively enrolled and compared with patients who underwent bronchoscopy with normal bronchoalveolar lavage (BAL) in the department of pulmonary medicine (control patients) [30].

\section{Bronchoalveolar lavage}

BAL was performed within 2 days of initiation of mechanical ventilation in ARDS patients, or in an outpatient setting for control participants. BAL fluid was obtained by centrifugation and cell population differentials were determined on cytospin slides after May-Grünwald-Giemsa staining.

\section{Cytokine quantification}

Interleukin (IL)-6, IL-8, CCL2, CXCL10, plasminogen activator inhibitor (PAI)-1 (ELISA Duoset; R\&D Systems, Abingdon, UK) and HMGB1 (ELISA; IBL International GmbH, Hamburg, Germany), were quantified in the BAL fluid by ELISA.

\section{Cell isolation and culture}

Human primary bronchial epithelial cells (BECs) were obtained from lung donor trachea or bronchi of the Cohort Of Lung Transplantation (COLT; trial registered at Clinicaltrials.gov, identifier NCT00980967). Tissues were dissociated overnight at $4^{\circ} \mathrm{C}$ with collagenase in HEPES-buffered RPMI medium (both from Sigma-Aldrich, St Louis, MO, USA). BECs were cultured in cnT17 medium (CELLnTEC Advanced Cell 
systems AG, Bern, Switzerland) containing penicillin and streptomycin, on plates coated with human type IV collagen (Sigma-Aldrich).

Blood samples were obtained from ARDS patients within the first hours following BAL, or from healthy donors. Neutrophils were purified as previously described [31]. Peripheral blood mononuclear cells (PBMCs) were isolated by Ficoll-Paque density gradient (Eurobio, Courtaboeuf, France). PBMCs were incubated in RPMI 1640 containing 7\% fetal calf serum (FCS) and $1 \%$ penicillin-streptomycin at $37^{\circ} \mathrm{C}$. After $1 \mathrm{~h}$, non-adherent cells were removed by washing with complete medium. Human monocyte-derived macrophages (HMDMs) were then derived from adherent monocytes by culture with $20 \mathrm{ng} \cdot \mathrm{mL}^{-1}$ macrophage colony-stimulating factor (M-CSF; R\&D Systems) for 5 days. Purity of HMDMs was $>80 \%$ and evaluated by flow cytometry.

\section{Apoptosis and necrosis assay}

BECs were cultured for $24 \mathrm{~h}$ in $50 \%$ cnT17 medium and 50\% BAL fluid or normal saline solution (Fresenius Kabi, Sèvres, France). BEC apoptosis and necrosis were assessed by flow cytometry using annexin V (Cell Signaling Technology, Danvers, MA, USA) and DAPI (4',6-diamidino-2-phenylindole; Life Technologies, Grand Island, NY, USA).

Circulating neutrophils purified from ARDS patients or healthy donors were cultured for $24 \mathrm{~h}$ in $50 \%$ RPMI/7\% FCS and 50\% BAL fluid or saline. Neutrophil apoptosis and necrosis were assessed by flow cytometry using a phycoerythrin-conjugated active caspase-3 apoptosis kit (Becton Dickinson, San Jose, CA, USA) and fluorescein isothiocyanate (FITC) anti-CD66b monoclonal antibody (mAb) (Beckman Coulter, Miami, FL, USA) for apoptosis. Annexin V and DAPI were used to measure necrosis.

\section{NET release quantification}

Neutrophils were incubated for $30 \mathrm{~min}$ in 50\% RPMI/7\% FCS and 50\% BAL fluid or saline. When indicated, BAL fluids were neutralised beforehand with an anti-HMGB1 mAb (IBL International GmbH) or isotype control for $2 \mathrm{~h}$. Neutrophils were then labelled with $5 \mu \mathrm{mol} \cdot \mathrm{L}^{-1}$ Sytox blue (Invitrogen, Carlsbad, CA, USA) in RPMI/0.5\% FCS with or without DNase I (200 IU.mL ${ }^{-1}$; Roche, Basel, Switzerland), seeded in Costar 96-well black plates (Corning Costar Corporation, Cambridge, MA, USA) and stimulated or not with $10 \mu \mathrm{mol} \cdot \mathrm{L}^{-1}$ phorbol myristate acetate (PMA; Sigma-Aldrich) for $3 \mathrm{~h}$ at $37^{\circ} \mathrm{C}$. The release of NETs (termed NETosis) was quantified by measuring fluorescence with a microplate fluorescence reader (Varioskan, ThermoFisher Scientific, Waltham, MA, USA).

\section{NET isolation and phagocytosis by macrophages}

Neutrophils from ARDS patients or healthy donors were incubated in RPMI with $25 \mathrm{nmol} \cdot \mathrm{L}^{-1}$ PMA for $2 \mathrm{~h}$ at $37^{\circ} \mathrm{C}$. After centrifugation, NETs were quantified in the supernatant by measuring fluorescence using Sytox blue $\left(5 \mu \mathrm{mol} \cdot \mathrm{L}^{-1}\right)$. HMDMs were allowed to attach in Costar 96 -well black plates for $3 \mathrm{~h}$ in $50 \%$ RPMI $/ 7 \%$ FCS and 50\% BAL fluid or saline, then Sytox blue-labelled purified NETs were added. After incubation for $2 \mathrm{~h}$ at $37^{\circ} \mathrm{C}$, HMDMs were washed and NET phagocytosis was assessed by fluorescence quantification. The NET engulfment ratio was determined as the ratio of fluorescence of HMDMs having phagocytised NETs to the fluorescence of HMDMs alone. When indicated, HMDMs were incubated with an anti-HMGB1 neutralising antibody or isotype control for $3 \mathrm{~h}$, or with metformin for $2.5 \mathrm{~h}\left(500 \mu \mathrm{mol} \cdot \mathrm{L}^{-1}\right.$; Sigma-Aldrich).

\section{Immunofluorescence stainings}

For NET imaging, purified neutrophils were immobilised on slides coated with poly-D-lysine (Sigma-Aldrich), and incubated with 50\% RPMI/7\% FCS and 50\% BAL fluid from control or ARDS patients for $3 \mathrm{~h}$. Cells were fixed with $4 \%$ paraformaldehyde (PFA; Antigenfix Diapath, Martingo, Italy). Coverslips were mounted with Mowiol including Sytox blue $\left(5 \mu \mathrm{mol} \cdot \mathrm{L}^{-1}\right)$.

For phagocytosis imaging, HMDMs were derived from monocytes on chamber coverslips with M-CSF (20 ng. $\mathrm{mL}^{-1}$ ) for 5 days. HMDMs were then incubated for $3 \mathrm{~h}$ with RPMI containing neutrophil-isolated NETs or not. Cells were fixed with $4 \%$ PFA and labelled with anti-neutrophil elastase mAb (Dako, Carpinteria, CA, USA) followed by Alexa Fluor 488 anti-mouse secondary antibody (Jackson ImmunoResearch, Ely, UK), and Texas Red-X Phalloidin (Life Technologies) for actin. Coverslips were mounted with Mowiol including TO-PRO-3 (1 $\mu \mathrm{mol} \cdot \mathrm{L}^{-1}$; Life Technologies).

For efferocytosis assays, HMDMs derived on chamber coverslips were incubated for $3 \mathrm{~h}$ with BAL fluid from control or ARDS patients, with or without $500 \mu \mathrm{mol} \cdot \mathrm{L}^{-1}$ metformin for $2.5 \mathrm{~h}$. When indicated, BAL fluid was pre-treated with an anti-HMGB1 neutralising antibody or isotype control. Efferocytosis was evaluated by adding $10^{6}$ carboxyfluorescein succinimidyl ester (CFSE)-labelled apoptotic neutrophils. After incubation at $37^{\circ} \mathrm{C}$ for $1 \mathrm{~h}$, cells were washed and fixed with $4 \%$ PFA. The efferocytosis index was 
determined on 300 cells as the percentage of HMDMs containing at least one ingested apoptotic neutrophil.

For all imaging, slides were examined with a SP5 confocal microscope (Leica Microsystems, Wetzlar, Germany). Digital images were processed using ImageJ software (National Institutes of Health, Bethesda, MD, USA).

\section{Western blot}

(Phospho)-AMPK Western blotting was performed using mouse anti-AMPK $\alpha$ or rabbit anti-phospho-AMPK $\alpha$ antibodies (Cell Signaling Technology), followed by horseradish peroxidase (HRP)-conjugated anti-mouse or anti-rabbit secondary IgG (Santa Cruz Biotechnology, Santa Cruz, CA, USA). Actin was blotted as loading control, using mouse anti- $\alpha$-actin (Sigma-Aldrich) and HRP-conjugated anti-mouse secondary IgG. Blots were quantified using ImageJ software.

\section{Statistical analysis}

Quantitative variables are expressed as mean \pm SD or median (interquartile range) when indicated, and qualitative variables as number (percentage). Continuous variables were compared using the nonparametric Mann-Whitney U-test or Wilcoxon test for matched pairs, as appropriate. Analyses were performed with GraphPad Prism 6.2 (GraphPad Software, La Jolla, CA, USA).

\section{Results}

NET formation in ARDS patients may contribute to lung injury

Among ARDS BAL leukocytes, neutrophils were the predominant cell population, whereas the majority of BAL leukocytes in controls are macrophages (online supplementary figure S1a and b). The characteristics of the ARDS patients and control subjects are provided in table 1 .

Several soluble factors implicated in the development of lung injury, including the pro-inflammatory cytokine IL-6, and CXCL10, CCL2 and IL-8 chemokines were significantly increased in ARDS patients (online supplementary figure S1c) [13, 32]. We also found significantly increased levels of PAI-1, implicated in downregulating efferocytosis in animal models of ALI (figure 1a) [33]. Because HMGB1 has been shown to promote NET release in experimental ALI, we also examined this possibility in ARDS patients [34]. We found that HMGB1 was significantly increased in BAL fluid of ARDS patients versus controls (figure 1b). Subsequent analysis revealed substantial amounts of cell-free DNA in the BAL fluid of ARDS patients, suggesting that HMGB1 accumulation is accompanied by an enhanced NETosis (figure 1c). Furthermore, BAL fluid from ARDS patients was found to induce lung epithelial cell injury, which could be related to NETs (figure $1 \mathrm{~d}-\mathrm{g}$ ).

Neutrophils of ARDS patients enhanced capacity to produce NETS

The cell-free DNA found in BAL fluid could be a result of DNA release from necrotic cell death. However, we found that neutrophils in BAL of ARDS patients had a relatively low apoptotic index (data not shown)

\section{TABLE 1 Patient characteristics}

\begin{tabular}{lcc} 
Characteristics & ARDS & Control \\
\hline Patients & 25 & 21 \\
Age years & $67(59-75)$ & $59(50-68)$ \\
Sex & & \\
$\quad$ Male & $14(56 \%)$ & $15(71 \%)$ \\
$\quad$ Female & $11(44 \%)$ & $6(28 \%)$ \\
Mortality & $7(28 \%)$ & $0(0 \%)$ \\
$\quad$ Dead & $18(72 \%)$ & $21(100 \%)$ \\
$\quad$ Alive & $22(88 \%)$ & NA \\
ARDS aetiology & $3(12 \%)$ & \\
$\quad$ Bacterial pneumonia & 3 & NA \\
Sepsis-related & $11(8-22)$ & NA \\
Influenza & $114(78-128)$ &
\end{tabular}

Data are presented as $n$, median (interquartile range) or $n(\%)$. ARDS: acute respiratory distress syndrome; $\mathrm{PaO}_{2}$ : arterial oxygen tension; $\mathrm{FlO}_{2}$ : inspiratory oxygen fraction; NA: not applicable. 
and also that circulating neutrophils of ARDS patients presented an increased capacity to produce NETs ex vivo, compared to healthy donors (figure $2 \mathrm{a}$ and $\mathrm{b}$ ). In these experiments, NETosis was measured after stimulation of neutrophils with PMA. NET formation was also used to examine whether BAL fluid from control or ARDS patients influences NET deployment. When compared to BAL fluid from control patients, BAL fluid from ARDS patients effectively increased spontaneous NET release from either control or ARDS neutrophils (figure 2c-f).
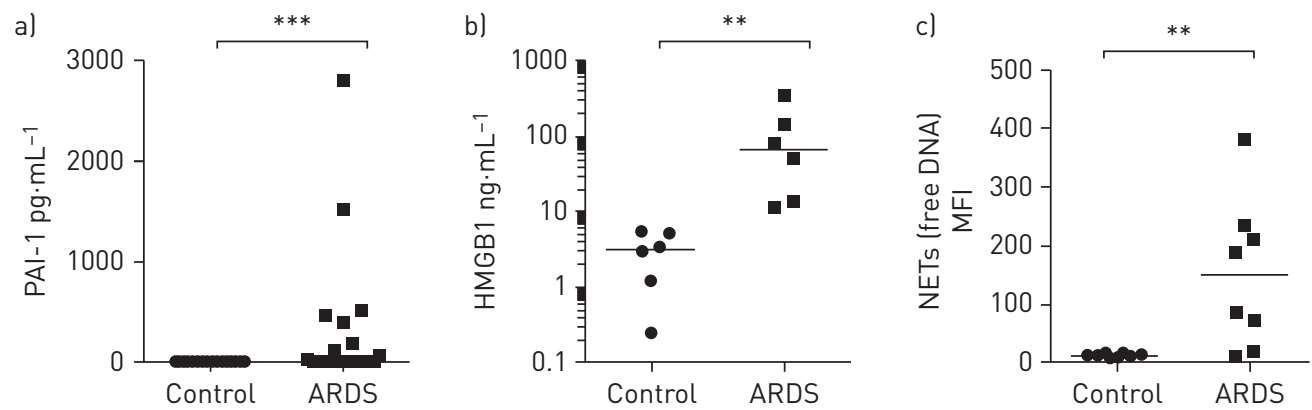

d)

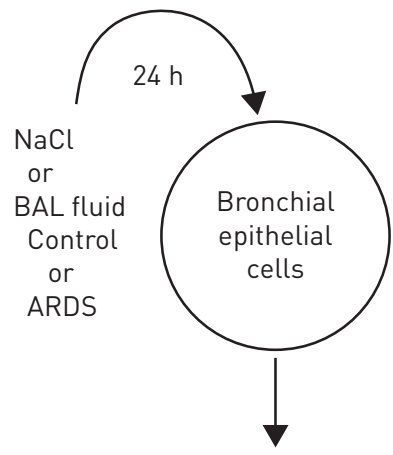

Apoptosis and necrosis

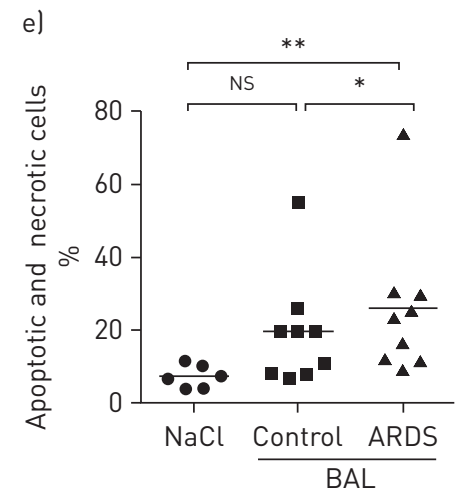

f)

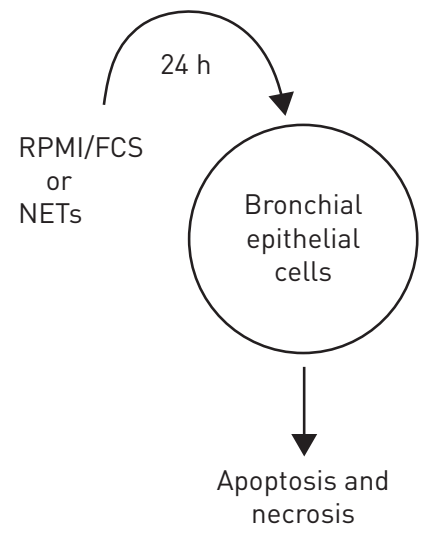

g)

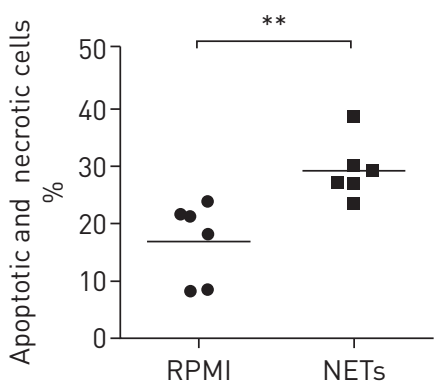

FIGURE 1 Characteristics of the bronchoalveolar lavage (BAL) fluid from acute respiratory distress syndrome (ARDS) patients may contribute to lung epithelial cell injury. a) Quantification of plasminogen activator inhibitor (PAI)-1 ( $n=19)$ and b) high-mobility group box 1 (HMGB1) ( $n=6)$ by ELISA in BAL fluid from control or ARDS patients. c) BAL fluid from ARDS patients contained high levels of neutrophil extracellular traps (NETs). Quantification of NETs by fluorescence measurement after Sytox blue staining in BAL fluid from control ( $\mathrm{n}=8$ ) and ARDS patients ( $n=8$ ). MFI: mean fluorescence intensity. $d$, e) BAL fluid from ARDS patients induced lung epithelial cell apoptosis and necrosis. d) Human primary bronchial epithelial cells (BECs) were treated with $50 \%$ normal saline solution ( $\mathrm{NaCl}$ ), or with BAL fluid from control or ARDS patients for $24 \mathrm{~h}$. e) Apoptosis and necrosis were measured using flow cytometry. $f, g)$ NETs induced lung epithelial cell apoptosis and necrosis. f) BECs were treated with $50 \%$ of RPMI/0.5\% fetal calf serum (FCS) or NETs for 24 h. g) Apoptosis and necrosis were measured using flow cytometry. In all graphs, horizontal bars represent medians. The MannWhitney U-test was used to compare protein and NET quantification and the Wilcoxon test was used for BEC apoptosis. ${ }^{*}: p<0.05 ;{ }^{* *}: p<0.01 ;{ }^{* *}$ : $p<0.001$; NS: nonsignificant. 
Neutrophils of ARDS patients show increased viability

In inflammatory conditions, like in ARDS, neutrophils are known to acquire a prolonged viability. To determine neutrophil viability, apoptotic indices were measured after $24 \mathrm{~h}$ of neutrophil culture. The amounts of apoptotic neutrophils were significantly lower in circulating neutrophils from ARDS patients compared to healthy donors. This result confirmed that viability of neutrophils is increased in ARDS patients (figure 3a). In subsequent experiments, we examined the effect of BAL fluids on neutrophil viability. As shown in figure $3 \mathrm{c}$, BAL fluid from ARDS patients, unlike that from control patients, increased the viability of healthy donor circulating neutrophils. The apoptotic percentage was even further decreased after exposure of ARDS circulating neutrophils to ARDS BAL fluid (figure 3d). However, to

a)

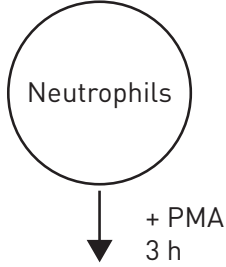

Free DNA quantification

c)

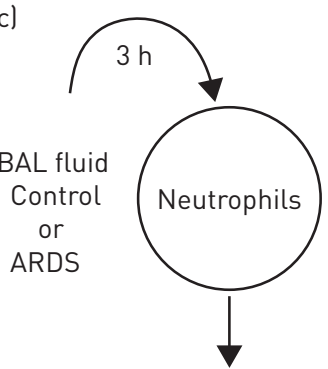

Free DNA quantification b)

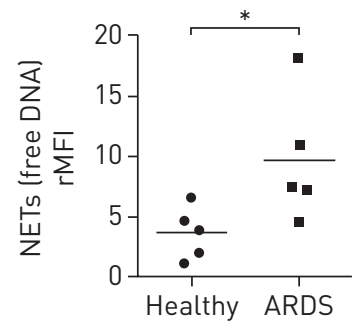

d)

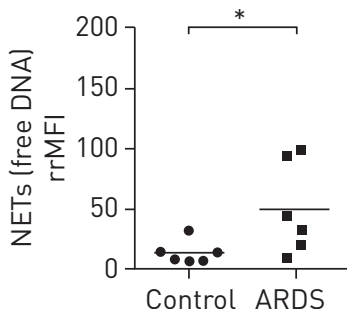

e)

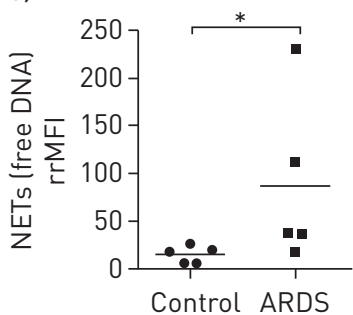

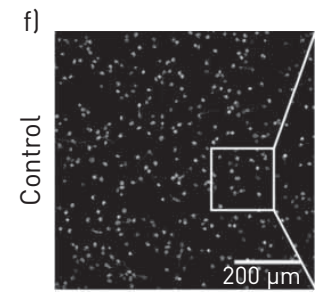
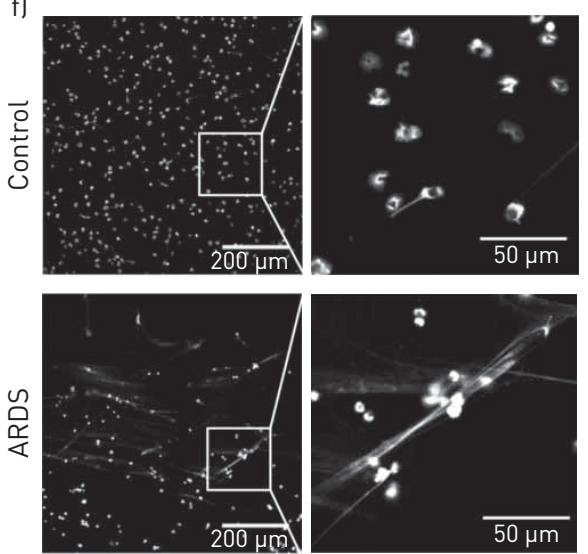

FIGURE 2 NETosis (release of neutrophil extracellular traps (NETs)) was enhanced in peripheral blood-derived neutrophils from acute respiratory distress syndrome (ARDS) patients, and increased by bronchoalveolar lavage (BAL) fluid mediators. a, b) Neutrophils from healthy donors or ARDS patients were treated with $10 \mu \mathrm{mol} \cdot \mathrm{L}^{-1}$ phorbol myristate acetate (PMA) for $3 \mathrm{~h}$ before NET quantification by Sytox blue fluorescence $\left(5 \mu \mathrm{mol} \cdot \mathrm{L}^{-1}\right)$. b) Free DNA (NETs) production was compared in neutrophils from healthy donors $(n=5)$ and ARDS patients $(n=5)$. rMFI: ratio of mean fluorescence intensity. c-e) Neutrophils from healthy donors or ARDS patients were treated with BAL fluid from control or ARDS patients for $3 \mathrm{~h}$ before NET quantification by Sytox blue fluorescence. rrMFI: ratio of ratio of mean fluorescence intensity. d) Quantification of NET production by healthy donor neutrophils after incubation with control or ARDS BAL fluid $(n=6)$. e) NET production by ARDS neutrophils was quantified after incubation with control or ARDS BAL fluid $(n=5)$. f) Fluorescence microscopy images showing NET formation from a representative ARDS patient after 3-h incubation with control or ARDS BAL fluid. Neutrophil DNA was stained with Sytox blue. In all graphs, horizontal bars represent medians. The Mann-Whitney U-test was used for comparisons. *: $\mathrm{p}<0.05$. 
ensure that specific constituent(s) of BAL fluid from ARDS patients increased neutrophil viability, we also explored necrosis rate and found a trend towards diminished neutrophil necrosis when exposed to BAL fluid from ARDS patients (figure 3e).

\section{HMDMs from ARDS patients have diminished ability to phagocytose NETs and apoptotic neutrophils}

The ability of macrophages to neutralise apoptotic neutrophils plays a central role in termination and resolution of inflammatory conditions. Recent studies also indicate that macrophages are involved in clearance of NETs $[20,35]$. As shown in figure $4 a-d$, there was a significant reduction in both NET uptake and apoptotic neutrophil efferocytosis by HMDMs from ARDS patients versus healthy donors. Moreover, a similar decrease in phagocytic ability was observed upon exposure of HMDMs from healthy donors to BAL fluid obtained from ARDS patients (figure 4e). Even further reduction in phagocytic index was found in ARDS HMDMs treated with ARDS BAL fluid (figure 4f). In contrast, BAL fluid from control patients had no impact on efferocytosis (figure $4 \mathrm{~d}$ versus $4 \mathrm{e}$ and $4 \mathrm{f}$ ). This finding also suggests that reduced efferocytosis was mediated by soluble components in the lung fluid of ARDS patients.

\section{The effects of AMPK and HMGB1 on efferocytosis and NET clearance}

To determine factor(s) that are affecting NETosis, efferocytosis and NET engulfment, we first examined the impact of HMGB1 (figure 5a). We did not observe any significant effect of HMGB1-neutralising antibody on NET formation (figure 5b). In contrast, we found that an anti-HMGB1 antibody increased the clearance of apoptotic neutrophils by ARDS HMDMs (figure 5c). However, it had no effect on NET

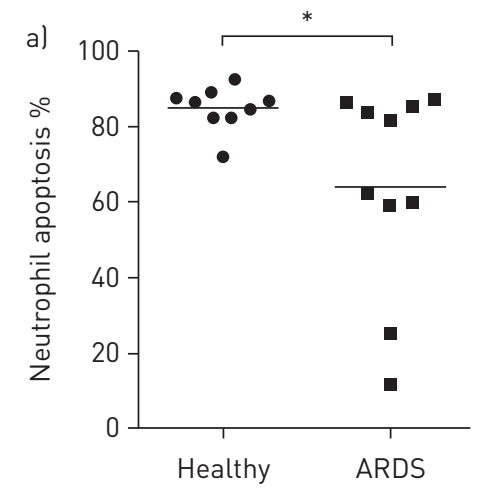

c)

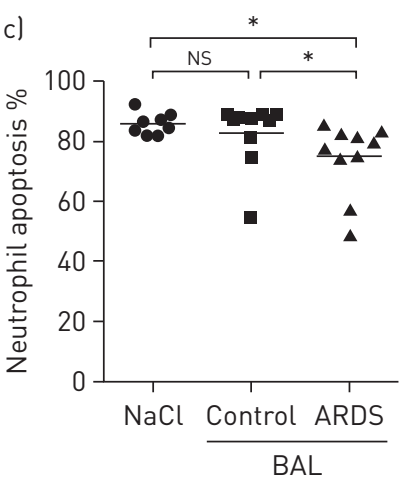

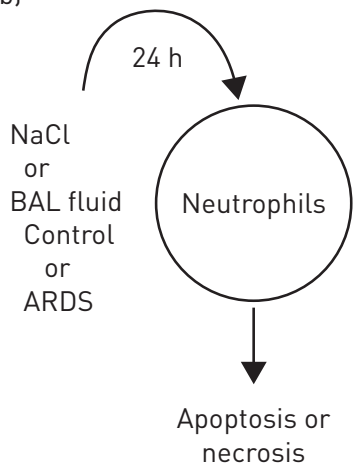

d)

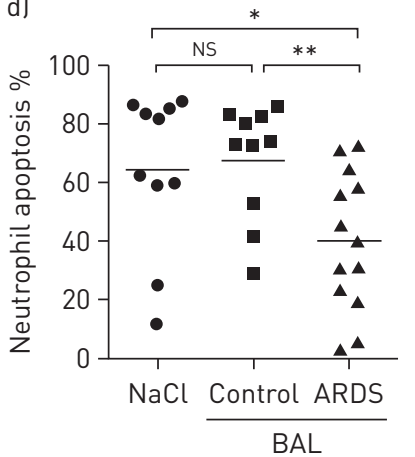

e)

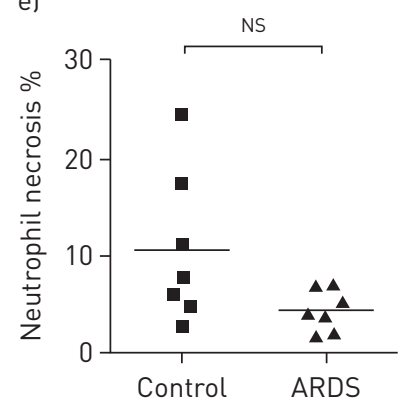

FIGURE 3 The proportion of apoptotic peripheral blood-derived neutrophils was decreased in acute respiratory distress syndrome (ARDS) patients, spontaneously and after incubation with bronchoalveolar lavage (BAL) fluid. a) Proportion of active caspase $-3^{+}$apoptotic cells among CD $66 b^{+}$neutrophils from healthy donors $(n=9)$ or ARDS patients $(n=10)$, assessed by flow cytometry after 24-h ex vivo culture. b) Neutrophils from healthy donors or ARDS patients were treated with $50 \%$ normal saline solution ( $\mathrm{NaCl}$ ), or with BAL fluid from control or ARDS patients for $24 \mathrm{~h}$ before neutrophil apoptosis or necrosis measurement. Proportion of active caspase $-3^{+}$apoptotic cells among $\mathrm{CD} 6 \mathrm{~b}^{+}$neutrophils from c) healthy donors, assessed by flow cytometry after 24-h ex vivo culture with $\mathrm{NaCl}(n=8)$, or control $(n=10)$ or ARDS ( $n=10)$ BAL fluid, and from d) ARDS patients, assessed by flow cytometry after 24-h ex vivo culture with $\mathrm{NaCl}(\mathrm{n}=10)$, or control ( $\mathrm{n}=10$ ) or ARDS ( $n=13$ ) BAL fluid. e) Proportion of annexin $\mathrm{V}^{+} / \mathrm{DAPI}^{+}$necrotic neutrophils from ARDS patients, assessed by flow cytometry after 24-h ex vivo culture with control (n=7) or ARDS ( $n=7)$ BAL fluid. In all graphs, horizontal bars represent medians. The Mann-Whitney U-test was used for comparisons. *: $p<0.05$; **: $p<0.01$; Ns: nonsignificant. 
uptake (figure 5d). The ability of HMGB1 to affect efferocytosis is consistent with previous studies in a murine model of inflammatory organ injury, in particular linking HMGB1 release into the extracellular milieu with diminished clearance of apoptotic cells [27-29].

Besides adverse effects mediated by extracellular HMGB1, inflammatory conditions are associated with metabolic reprogramming of immune and parenchymal cells that is associated with diminished activity of AMPK in macrophages [36]. Notably, AMPK activators, including metformin, have been shown to promote efferocytosis and also to reduce the severity of ALI [24-26]. Thus, we examined whether AMPK activation can also recover the phagocytic capacity of HMDMs from ARDS patients. As shown in

a)

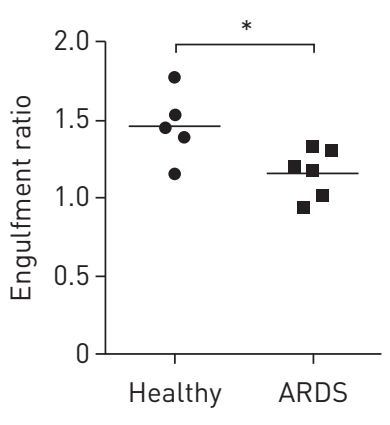

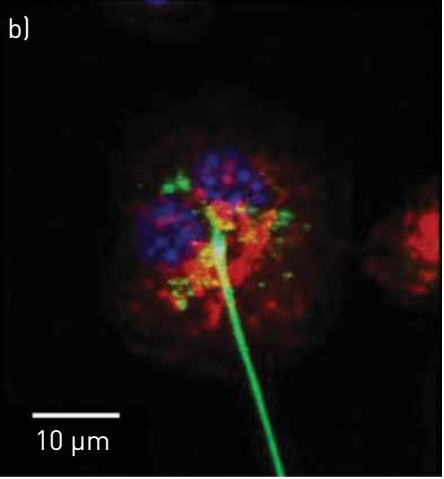

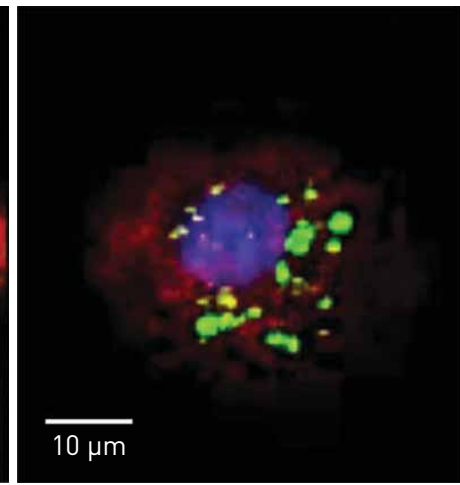

c)

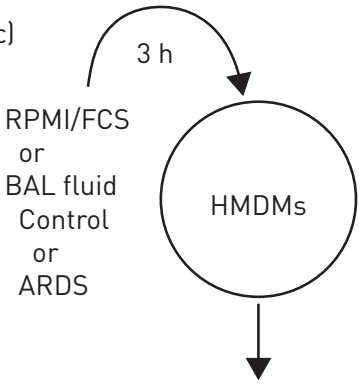

Efferocytosis

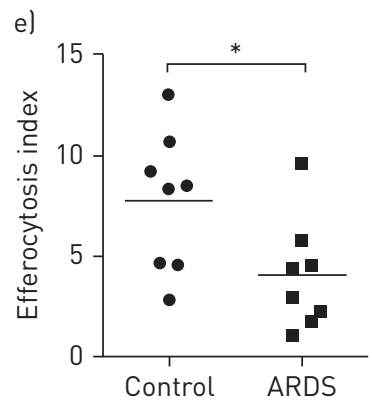

d)

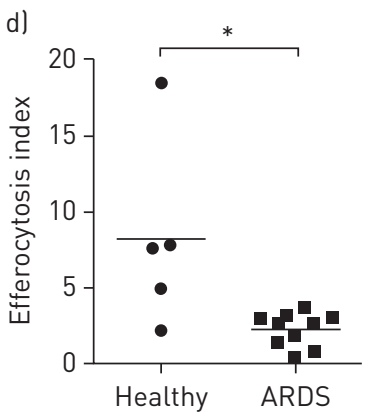

f)

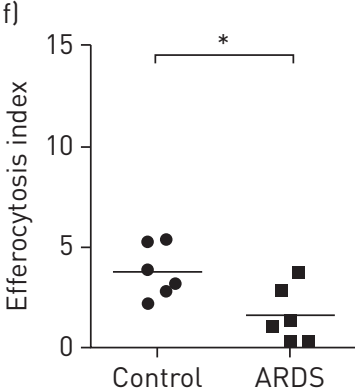

FIGURE 4 The ability of human monocyte-derived macrophages (HMDMs) to engulf neutrophil extracellular traps (NETs) and apoptotic neutrophils was reduced during acute respiratory distress syndrome (ARDS). a) Engulfment of Sytox blue-labelled NETs by HMDMs from healthy donors $(n=6)$ and ARDS patients $(n=6)$. The NET engulfment ratio was defined as the ratio of fluorescence emitted by HMDMs that had phagocytosed NETs to the fluorescence emitted by HMDMs alone. b) Immunofluorescence images showing HMDMs in the process of engulfing NETs. HMDMs were incubated with NETs purified from neutrophils and these were internalised by HMDMs. The HMDM actin was stained with phalloidin (red), DNA with Sytox blue (blue), and NET neutrophil elastase with antibodies (green). c-f) The efferocytosis capacities of HMDMs were decreased by ARDS, both spontaneously and after incubation with bronchoalveolar lavage (BAL) fluid. c) HMDMs were incubated with RPMI/fetal calf serum (FCS) or BAL fluid for $3 \mathrm{~h}$ before adding carboxyfluorescein succinimidyl ester (CFSE)-labelled apoptotic neutrophils for $1 \mathrm{~h}$. The efferocytosis index was defined as the number of HMDMs that phagocytosed apoptotic neutrophils relative to the number of HMDMs that did not. Efferocytosis index of d) HMDMs from healthy donors ( $n=5)$ versus ARDS patients $(n=10)$, e) healthy donor macrophages cultured with control $(n=8)$ or ARDS ( $n=8)$ BAL fluid, and f) macrophages from ARDS patients cultured with control ( $n=8)$ or ARDS ( $n=8)$ BAL fluid. In all graphs, horizontal bars represent medians. The Mann-Whitney U-test was used for comparisons. * $p<0.05$. 
a)

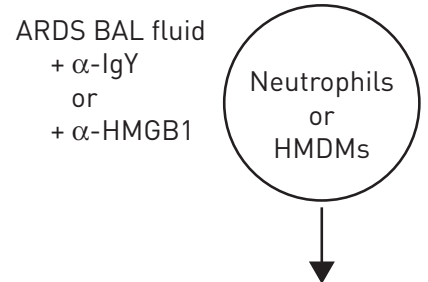

Free DNA

quantification,

efferocytosis and

NET engulfment b)

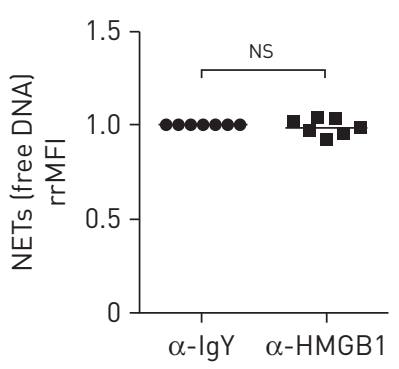

c

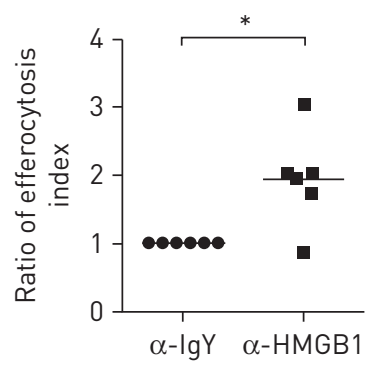

d)

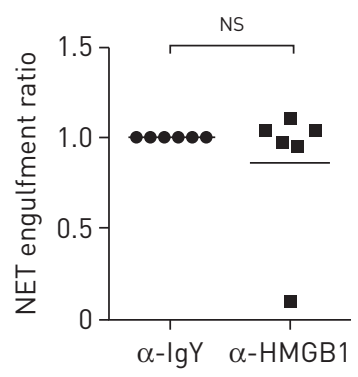

e)

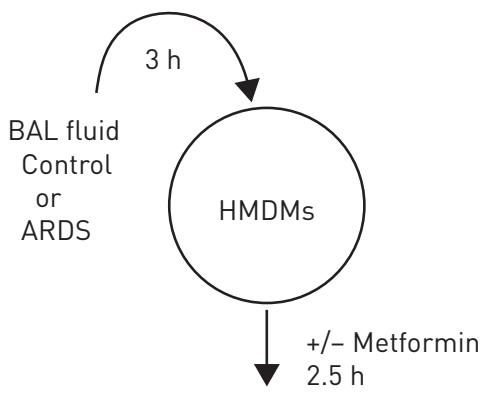

AMPK activity, efferocytosis and NET engulfment f)
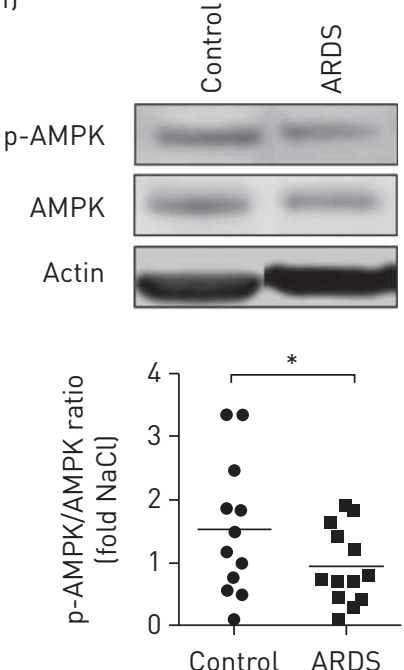

g)

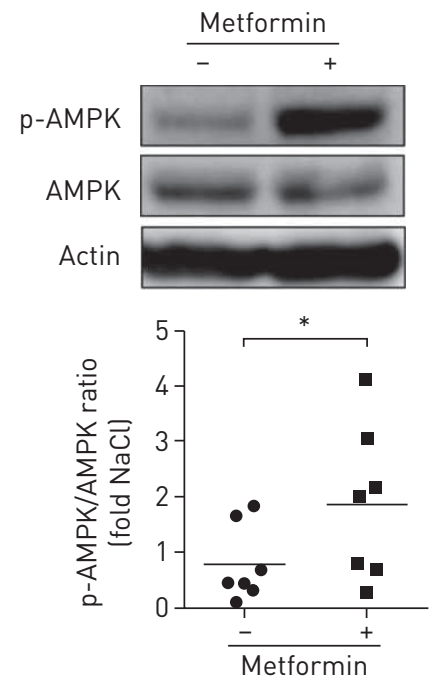

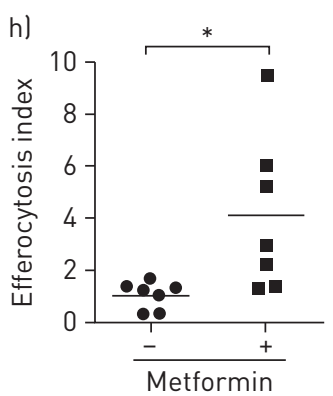

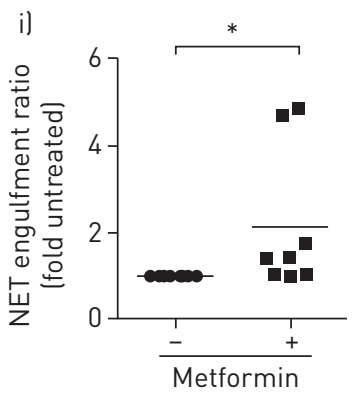

FIGURE 5 Inhibition of high-mobility group box 1 (HMGB1) and activation of AMP-activated protein kinase (AMPK) increased neutrophil extracellular trap (NET) engulfment and efferocytosis by human monocyte-derived macrophages (HMDMs). a) Bronchoalveolar lavage (BAL) fluid from acute respiratory distress syndrome (ARDS) patients was treated for $2 \mathrm{~h}$ with an anti-HMGB1 ( $\alpha$-HMGB1) or isotype control antibody ( $\alpha$-IgY) before incubation with neutrophils from healthy donors or HMDMs from ARDS patients for $3 \mathrm{~h}$. b) NET production by healthy donor neutrophils $(n=8)$. rrMFI: ratio of ratio of mean fluorescence intensity. c) HMDM efferocytosis index was determined after 1-h contact with apoptotic neutrophils $(n=6)$. The efferocytosis index was defined as the number of HMDMs that phagocytosed apoptotic neutrophils relative to the number of HMDMs that did not. d) NET engulfment by HMDMs from ARDS patients $(n=6)$ was determined after 2-h contact with neutrophil-derived NETs. The NET engulfment ratio was defined as the ratio of fluorescence emitted by HMDMs that had phagocytosed NETs to the fluorescence emitted by HMDMs alone. e) HMDMs from ARDS patients were incubated with BAL fluid from control or ARDS patients for $3 \mathrm{~h}$ before being treated with or without metformin for $2.5 \mathrm{~h}$. Representative Western blots and quantitative analysis of phospho (p)-AMPK, total AMPK and actin from ARDS patient HMDMs incubated with f) control or ARDS BAL fluid, and with g) ARDS BAL fluid and metformin or medium alone. h) HMDM efferocytosis index determined after 1 - $\mathrm{h}$ contact with apoptotic neutrophils $(\mathrm{n}=7)$. i) Engulfment by HMDMs of Sytox blue-stained NETs, determined after $2 \mathrm{~h}$ (n=8). In all graphs, horizontal bars represent medians. The Wilcoxon test was used to compare effects of different HMGB1 or metformin treatments on NET engulfment and efferocytosis. *: $p<0.05$; NS: nonsignificant. 
figure 5f, AMPK activation, i.e. phosphorylation of Thr172-AMPK, was significantly diminished in HMDMs treated with BAL fluid from ARDS patients. Moreover, we found that culture of ARDS HMDMs with metformin restored AMPK activation (figure $5 \mathrm{~g}$ ). This activation was associated with a significant increase in uptake of apoptotic neutrophils and NETs (figure $5 \mathrm{~h}$ and i).

\section{Discussion}

Our study reveals major findings that could enhance or sustain lung inflammation during ARDS. First, although neutrophil lifespan is significantly increased, intra-alveolar neutrophils are releasing NETs through a pathway termed vital NETosis and BAL fluid of ARDS patients can increase the release of NETs, which could induce lung injury. Secondly, in ARDS conditions, the ability of macrophages to engulf NETs and apoptotic cells is significantly decreased. We also found that blocking HMGB1 and activating AMPK could enhance clearance of NETs and apoptotic cells.

Clinical and histological studies have suggested that the severity and outcome of ARDS were associated with the inflammatory process reflected in bronchoalveolar fluid [13]. A large number of clinical and animal studies have brought evidence that neutrophils have a direct influence on the onset and the persistence of ARDS. For instance, STEINBERG et al. [12] found that alveolar macrophages increased in ARDS survivors compared to nonsurvivors, and reached the conclusion that sustained alveolar inflammation was associated with high mortality. Among factors that could sustain inflammatory conditions, increased NETosis and decreased ability of macrophages to engulf apoptotic cells and NETs appear to be critical.

We found that neutrophils could produce large amounts of NETs, spontaneously or when exposed to ARDS BAL fluid. NETs are composed of decondensed chromatin fibres coated with antimicrobial proteins. NETosis could require membrane rupture and the loss of neutrophil functions (so-called "suicidal NETosis") [17]. However, YIPP and KubES [37] have demonstrated that, during the early phase of infection, NETosis involved neutrophils that did not undergo lysis and retained the ability to perform recruitment, chemotaxis and phagocytosis (so-called "vital NETosis"). In our study, and probably in the ARDS setting, NETosis does not result in cell death, since we found that neutrophil lifespan was increased. Although the primary role of NETs is to avoid bacterial diffusion, NETs have been found to play deleterious effects on lung injury during ARDS and several studies have pointed out that NET formation during bacterial pneumonia only worsened lung injury, without any bactericidal activity [17, 38]. Moreover, NARASARAju et al. [15] demonstrated that, in mice challenged with influenza, NETs contributed to ALI by instigating alveolar capillary damage. Therefore, NETosis and clearance of NETs should be adequately regulated in vivo and defects in mechanisms responsible for NET clearance may contribute to perpetuated inflammation and worsening of tissue injury $[15,39]$. Two mechanisms have been described in NET clearance: DNase I-dependent digestion and phagocytosis by macrophages [17]. The latter has been found to be diminished in our study. We also demonstrated that efferocytosis was decreased in ARDS. Clearance of cells undergoing apoptosis protects surrounding tissue from exposure to pro-inflammatory intracellular contents released from necrotic cells. Although there are convincing animal data showing that neutrophils secrete anti-inflammatory peptides while dying, failure to effectively remove apoptotic cells (particularly apoptotic neutrophils) perpetuates inflammation, exposing the lung to sustained inflammatory conditions that could increase neutrophil influx and NETosis and worsen lung injury $[40,41]$. Thus, macrophages have a key position in the resolution of inflammation and initiation of tissue repair. Restoring the ability of macrophages to engulf both NETs and apoptotic cells could be of interest to decrease lung damage and pulmonary sequelae such as fibrosis.

We found that BAL fluid from ARDS patients could induce a decrease in efferocytosis in both healthy and ARDS macrophages, creating the possibility that therapeutic interventions could enhance efferocytosis and engulfment of NETs. Along these lines, the AMPK pathway appears to be a potential therapeutic target. We found in our study that the ability of macrophages to activate AMPK was decreased in inflammatory conditions, which could be associated with a defect in efferocytosis activity [24]. Notably, the ability of AMPK activation to enhance phagocytosis appears to be related to interaction with cytoskeletal organisation [24]. Lastly, we found that inhibition of HMGB1 could increase efferocytosis, as already reported in animal models of lung injury. HMGB1, originally described as a nuclear nonhistone DNA-binding protein, has subsequently been shown to be an alarmin involved in the inflammatory response playing a critical role in the recruitment of neutrophils, lung injury and suppressing bacterial clearance in the lung $[31,34,42]$. It is worth noting that metformin has been found to bind and inhibit the action of HMGB1, suggesting that the effects of metformin could be similar to those of HMGB1 inhibition [43]. High levels of HMGB1 in BAL fluid from ARDS patients can decrease efferocytosis, suggesting that inhibition of HMGB1, as well as activation of AMPK, could be of interest to restore efferocytosis, diminish lung injuries and ultimately improve lung function after ICU discharge. 


\section{Conclusion}

Altogether, our results show that efferocytosis and NET engulfment, which could contribute to the persistence of lung inflammation, are diminished during ARDS. Restoration of AMPK activation with metformin and specific inhibition of HMGB1 appear to be promising targets to decrease lung inflammation and to limit alveolar damage and progression to lung fibrosis in patients with ARDS.

Acknowledgements: Immunofluorescence study was performed at the Microscopy Rennes Imaging Center (MRic-ALMF; SFR UMS CNRS 3480 - INSERM 018 Biosit, Rennes, France). Healthy donor monocytes were provided from the DTC Center (CIC Biothérapie 0503, Nantes, France). The authors thank Mohinder Pal (University of Sussex, Genome Center, Brighton, UK) for the careful reading of the manuscript.

Conflict of interest: None declared.

Support statement: This work was supported by grants from CORECT 2015 (Comité de la Recherche Clinique et Translationnelle), CHU Rennes, France. Funding information for this article has been deposited with the Crossref Funder Registry.

\section{References}

1 Thille AW, Esteban A, Fernández-Segoviano P, et al. Comparison of the Berlin definition for acute respiratory distress syndrome with autopsy. Am J Respir Crit Care Med 2013; 187: 761-767.

2 Burnham EL, Janssen WJ, Riches DW, et al. The fibroproliferative response in acute respiratory distress syndrome: mechanisms and clinical significance. Eur Respir J 2014; 43: 276-285.

3 Papazian L, Forel JM, Gacouin A, et al. Neuromuscular blockers in early acute respiratory distress syndrome. N Engl J Med 2010; 363: 1107-1116.

4 Guérin C, Reignier J, Richard JC, et al. Prone positioning in severe acute respiratory distress syndrome. $N$ Engl J Med 2013; 368: 2159-2168.

5 Ward PA. New strategies for treatment of humans with acute lung injury/acute respiratory distress syndrome. Clin Infect Dis 2015; 60: 596-597.

6 Kolaczkowska E, Kubes P. Neutrophil recruitment and function in health and inflammation. Nat Rev Immunol 2013; 13: 159-175

7 Brinkmann V, Reichard U, Goosmann C, et al. Neutrophil extracellular traps kill bacteria. Science 2004; 303: 1532-1535.

8 Yipp BG, Petri B, Salina D, et al. Infection-induced NETosis is a dynamic process involving neutrophil multitasking in vivo. Nat Med 2012; 18: 1386-1393.

9 Abraham E. What role does neutrophil apoptosis play in acute respiratory distress syndrome? Crit Care Med 2000; 28: $253-254$.

10 Grommes J, Soehnlein O. Contribution of neutrophils to acute lung injury. Mol Med 2011; 17: 293-307.

11 Williams AE, Chambers RC. The mercurial nature of neutrophils: still an enigma in ARDS? Am J Physiol Lung Cell Mol Physiol 2014; 306: L217-L230.

12 Steinberg KP, Milberg JA, Martin TR, et al. Evolution of bronchoalveolar cell populations in the adult respiratory distress syndrome. Am J Respir Crit Care Med 1994; 150: 113-122.

13 Goodman RB, Strieter RM, Martin DP, et al. Inflammatory cytokines in patients with persistence of the acute respiratory distress syndrome. Am J Respir Crit Care Med 1996; 154: 602-611.

14 Chiara AD, Pederzoli-Ribeil M, Burgel PR, et al. Targeting cytosolic proliferating cell nuclear antigen in neutrophil-dominated inflammation. Front Immunol 2012; 3: 311.

15 Narasaraju T, Yang E, Samy RP, et al. Excessive neutrophils and neutrophil extracellular traps contribute to acute lung injury of influenza pneumonitis. Am J Pathol 2011; 179: 199-210.

16 Kaplan MJ, Radic M. Neutrophil extracellular traps: double-edged swords of innate immunity. J Immunol 2012; 189: 2689-2695.

17 Sørensen OE, Borregaard N. Neutrophil extracellular traps - the dark side of neutrophils. J Clin Invest 2016; 126: $1612-1620$

18 Mahajan A, Herrmann M, Muñoz LE. Clearance deficiency and cell death pathways: a model for the pathogenesis of SLE. Front Immunol 2016; 7: 35.

19 Wootton DG, Diggle PJ, Court J, et al. Recovery from pneumonia requires efferocytosis which is impaired in smokers and those with low body mass index and enhanced by statins. Thorax 2016; 71: 1052-1054.

20 Nakazawa D, Shida H, Kusunoki Y, et al. The responses of macrophages in interaction with neutrophils that undergo NETosis. J Autoimmun 2016; 67: 19-28.

21 Byrne AJ, Mathie SA, Gregory LG, et al. Pulmonary macrophages: key players in the innate defence of the airways. Thorax 2015; 70: 1189-1196.

22 Farrera C, Fadeel B. Macrophage clearance of neutrophil extracellular traps is a silent process. J Immunol 2013; 191: $2647-2656$

23 Lin SC, Hardie DG. AMPK: sensing glucose as well as cellular energy status. Cell Metab 2018; 27: 299-313.

24 Bae HB, Zmijewski JW, Deshane JS, et al. AMP-activated protein kinase enhances the phagocytic ability of macrophages and neutrophils. FASEB J 2011; 25: 4358-4368.

25 Park DW, Jiang S, Tadie JM, et al. Activation of AMPK enhances neutrophil chemotaxis and bacterial killing. Mol Med 2013; 19: 387-398.

26 Park DW, Jiang S, Liu Y, et al. GSK3ß-dependent inhibition of AMPK potentiates activation of neutrophils and macrophages and enhances severity of acute lung injury. Am J Physiol Lung Cell Mol Physiol 2014; 307: L735-L745.

27 Liu G, Wang J, Park YJ, et al. High mobility group protein-1 inhibits phagocytosis of apoptotic neutrophils through binding to phosphatidylserine. J Immunol 2008; 181: 4240-4246. 
28 Friggeri A, Yang Y, Banerjee S, et al. HMGB1 inhibits macrophage activity in efferocytosis through binding to the $\alpha_{v} \beta_{3}$-integrin. Am J Physiol Cell Physiol 2010; 299: C1267-C1276.

29 Banerjee S, de Freitas A, Friggeri A, et al. Intracellular HMGB1 negatively regulates efferocytosis. J Immunol 2011; 187: 4686-4694.

30 Thille AW, Vuylsteke A, Bersten A. Does the Berlin definition for acute respiratory distress syndrome predict the presence of diffuse alveolar damage? Intensive Care Med 2015; 41: 342-344.

31 Grégoire M, Tadié JM, Uhel F, et al. HMGB1 induces neutrophil dysfunction in experimental sepsis and in patients who survive septic shock. J Leukoc Biol 2017; 101: 1281-1287.

32 Michalec L, Choudhury BK, Postlethwait E, et al. CCL7 and CXCL10 orchestrate oxidative stress-induced neutrophilic lung inflammation. J Immunol 2002; 168: 846-852.

33 Park YJ, Liu G, Lorne EF, et al. PAI-1 inhibits neutrophil efferocytosis. Proc Natl Acad Sci USA 2008; 105: 11784-11789.

34 Tadie JM, Bae HB, Jiang S, et al. HMGB1 promotes neutrophil extracellular trap formation through interactions with Toll-like receptor 4. Am J Physiol Lung Cell Mol Physiol 2013; 304: L342-L349.

35 Boe DM, Curtis BJ, Chen MM, et al. Extracellular traps and macrophages: new roles for the versatile phagocyte. J Leukoc Biol 2015; 97: 1023-1035.

36 Tadie JM, Bae HB, Deshane JS, et al. Toll-like receptor 4 engagement inhibits adenosine 5'-monophosphate-activated protein kinase activation through a high mobility group box 1 protein-dependent mechanism. Mol Med 2012; 18: 659-668.

37 Yipp BG, Kubes P. NETosis: how vital is it? Blood 2013; 122: 2784-2794.

38 Porto BN, Stein RT. Neutrophil extracellular traps in pulmonary diseases: too much of a good thing? Front Immunol 2016; 7: 311

39 Müller-Redetzky H. Targeting neutrophil extracellular traps in acute lung injury: a novel therapeutic approach in acute respiratory distress syndrome? Anesthesiology 2015; 122: 725-727.

40 McCubbrey AL, Curtis JL. Efferocytosis and lung disease. Chest 2013; 143: 1750-1757.

41 Miles K, Clarke DJ, Lu W, et al. Dying and necrotic neutrophils are anti-inflammatory secondary to the release of $\alpha$-defensins. J Immunol 2009; 183: 2122-2132.

42 Lotze MT, Tracey KJ. High-mobility group box 1 protein (HMGB1): nuclear weapon in the immune arsenal. Nat Rev Immunol 2005; 5: 331-342.

43 Horiuchi T, Sakata N, Narumi Y, et al. Metformin directly binds the alarmin HMGB1 and inhibits its proinflammatory activity. J Biol Chem 2017; 292: 8436-8446. 